\title{
Trial Production of Modified Tricopter Based Vertical Take-off Landing Canard Aircraft with Tilt Tail Rotor
}

\author{
Kiyoteru Hayama ${ }^{\mathrm{a}, *}$, Tomohiro Kudou ${ }^{\mathrm{b}}$, and Hiroki Irie ${ }^{\mathrm{a}}$ \\ ${ }^{a}$ Fuculty of Project Centers, ${ }^{b}$ Fuculty of Liberal Studies, \\ National Institute of Technology, Kumamoto College, \\ 2659-2 Suya, Koshi, Kumamoto 861-1102, Japan \\ *Corresponding Author: hayama@kumamoto-nct.ac.jp
}

\begin{abstract}
The trial production of a new concept vertical take-off and landing (VTOL) canard aircraft based on the modified tricopter with tilt tail rotor was carried out for aerial, observation and research. Continuous transition from vertical to horizontal flight can be done by tilting the tail rotor supported with canard wing. The lift of wing during horizontal flight supported the weight of the aircraft, and its causes the reduction of power consumption and extend the flight area.
\end{abstract}

Keywords: VTOL, canard wing, multicopter, tricopter, tilt rotor, power saving.

\section{Introduction}

The multi-rotor helicopter also called multicopter is widely spread for the less mechanical parts, electrically controllable and small space of the take-off and landing, and is useful for aerial, observation and research applications (1). However, the time-of-flight and cruising distance is limited by the battery capacity. On the other hand, though the fixed-wing aircraft has an advantage of wide range of flight area caused by the high-speed and long-time flight of energy efficiency than the rotorcraft, it cannot hover in the air and takes a runway field to take-off and landing. Assuming the exploration and observation at disaster sites, it is difficult to secure the airfield, therefore, a new flying machine is desired that has both feature of the rotorcraft for vertical take-off and landing (VTOL) and fixed-wing aircraft for wide range of flight. There are many kind of VTOL ${ }^{(2)}$. It is well known that the tilt rotor system and tail-sitter aircraft. The tail-sitter takes off and land on its tail, then tilts horizontally for forward flight ${ }^{(3)}$. The tilt rotor system can be vertical and horizontal flight by changing the angle of rotors ${ }^{(4)}$. However, attitude control for both aircraft is difficult in transition flight to vertical and horizontal at the time of take-off and landing.

Recently, the wingcopter ${ }^{(5)}$ is well known as highly stable VTOL with a tilt rotors, whch is hover with quad rotors, and transition to level flight with all tilting rotors. The quadplane $^{(6)}$ is also known as a open source VTOL project, which is equipped with quadrotors on an airplane. The disadvantages of these are that there are more components than an airplane, and the weight of the aircraft increases.

In our previous research, trial production of variable pitch wing attached multicopter is carried out. It was confirmed that the lift of the wing during level flight is partially supported the weight of the aircraft and is effective to reduce the power consumption ${ }^{(7)}$. However, these aircraft consume more power than airplanes, and further improvement of efficiency is desired.

In this study, we propose a new concept VTOL canard aircraft based on the modified tricopter with tilt tail rotor to earn the advantage for energy saving of wing. We aim for highly efficient flight with a complete conversion to an airplane.

\section{Proposed Rotorcraft}

\subsection{Comparison with our previous studies}

Figure 1 shows our previous research of variable pitch wing attached multicopter ${ }^{(7)}$. Continuous transition from rotorcraft to like fixed-wing aircraft could be done with adjusting suitable angle of attack of the wing of the rotorcraft. It was confirmed that the lift of the wing during 


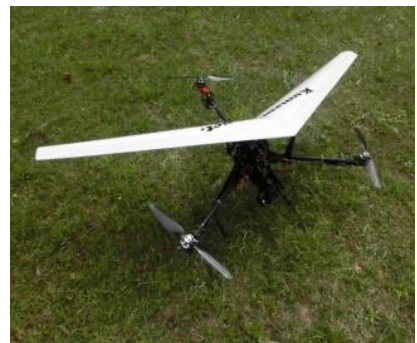

Fig. 1. Y3 tricopter with variable pitch wing.

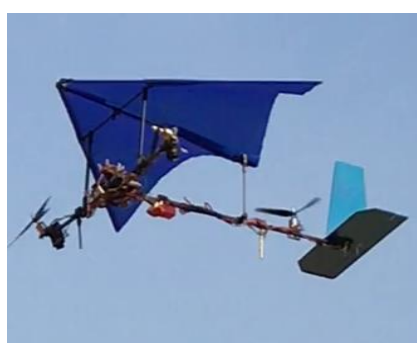

Fig. 2. Flexible kite wing attached tricoated with tilt front rotors. horizontal flight was effective to reduce the power consumption less than $66 \%$ compared with that without wing, and the flight area could be extended widely. However, disadvantages of the rotorcraft are also derived. The rotorcraft is difficult to climb and descend rapidly. Lift of the wing cannot be obtained except for forward flight. It is investigated the main wing is affected by side wind and turbulence generated by own rotors, which is negative factor in stability during take-off and landing of the rotorcraft. The rotorcraft has poor portability because dividing the main wing has a limitation for the aspect of securing its strength, in contrast to the multicopter can improve portability by folding the rod mounting rotors.

The flexible kite wing attached tricopter with tilt front rotors shown in Fig. 2 was also developed to earn the advantage of the attachment of variable pitch wing ${ }^{(8)}$. The kite wing has much tolerance for the pitch angle. The wing area and mounting angle can be pre-fixed. The flexible wing can be solved portability of the wing and avoiding the bad influence of turbulence by rotors during take-off and landing. It was confirmed that the complete conversion to airplane by $90^{\circ}$ tilt of front rotors and lift of the kite wing during horizontal flight can be reduced the power consumption less than $70 \%$ compared with that during hovering. However, the lift-to-drag ratio of the kite is not large, and the power saving effect is small.

\subsection{Principle of the proposed aircraft}

Figure 3 shows our new concept VTOL canard aircraft based on the modified tricopter with tilt tail rotor. We aim for highly efficient flight with a complete conversion to an airplane. We have designed the aircraft based on tricopter which is consists of the minimum number of three rotors

Table 1. Comparison of components of VTOL aircrafts.

\begin{tabular}{|c|c|c|c|c|c|c|}
\hline \multirow{2}{*}{ Component } & \multicolumn{2}{|c|}{ QuadPlane } & \multicolumn{2}{c|}{ WingCopter } & \multicolumn{2}{c|}{ Proposed aircraft } \\
\cline { 2 - 7 } & Hovering & Levelflight & Hovering & Levelflight & Hovering & Levelflight \\
\hline Motors & 5 & 1 & 4 & 4 & 3 & 1 \\
\hline Tilt servos & 0 & 0 & 0 & 4 & 0 & 1 \\
\hline Controlservos & 0 & 2 or 4 & 0 & 2 or 4 & 1 & 2 \\
\hline
\end{tabular}

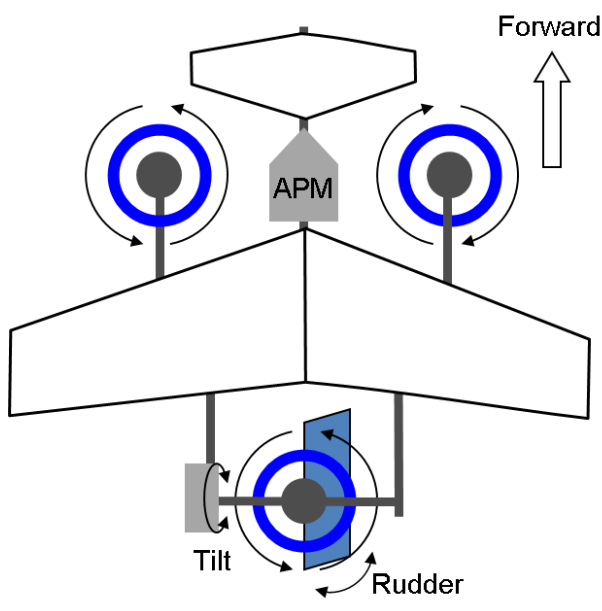

Fig. 3. Concept of the proposed aircraft

required for hovering and attitude stabilization. Whereas a normal tricopter tilts the tail rotor, the rudder instead cancels the counter torque of tail rotor. The aircraft has a main wing for lift and a canard wing to stabilize the pitching. The center of gravity of the canard aircraft is designed corresponding to the combined thrust force of tricopter. This pushing thrust allows transition to the horizontal flight.

Table 1 shows a comparison the component of motors, tilt servos and control servos at different type of VTOL aircrafts. Our proposed aircraft has minimum configuration during both hovering and level flight and is effective reducing the weight of the aircraft.

Figure 4 shows the weight and balance acting on the aircraft during flight. During hovering, the flight controller (FC) adjusts the thrust of three rotors to keep the balance of the aircraft as shown in fig.4(a). By tilting tail rotor gently, the aircraft can smoothly transition to level flight (fig. 4(b)).
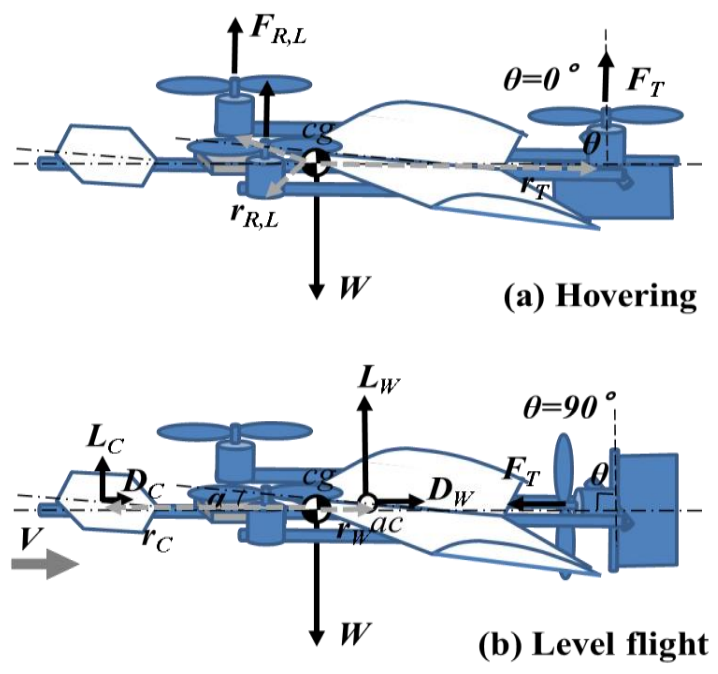

Fig. 4. Weight and balance acting on the aircraft. 
When level flight, the lift of main and canard wings $\left(\mathrm{L}_{\mathrm{w}}\right.$, $\mathrm{L}_{\mathrm{C}}$ ) and weight of the aircraft $(\mathrm{W})$ are balanced, also the thrust of tail rotor $\left(\mathrm{F}_{\mathrm{T}}\right)$ and drags $\left(\mathrm{D}_{\mathrm{W}}, \mathrm{D}_{\mathrm{C}}\right)$ are balanced. So, the front rotors become unnecessary and further power saving is achieved.

\section{Trial production of the proposed aircraft}

Figure 5 shows trial production of the proposed aircraft. The base flame is made by carbon and duralumin rods mounted three blushless motors. The maximum thrust force and power consumption of the motor using 3 blade 5 inches propeller are $0.44 \mathrm{~kg}$ and $138 \mathrm{~W}$ each, respectively. There is enough thrust to lift the aircraft with three rotors. The total weight including LiPo battery (3S-11.1V, 1400mAh) was about $0.66 \mathrm{~kg}$. The wingspan is $0.9 \mathrm{~m}$, and the wing area is $0.22 \mathrm{~m}^{2}$.

Figure 6 shows the tilt of tail rotor and rudder. As shown in Fig. 6(a) for hovering, the aircraft rotates on the yaw axis by rudder operation. When level flight, the tail rotor is tilted forward as shown in Fig. 6(b). The rudder also works same as that of airplane.

Figure 7 shows the block diagram of the proposed aircraft. The aircraft is operated by $2.4 \mathrm{GHz} \mathrm{R} / \mathrm{C}$ system. We have used the CC3D ${ }^{(9)}$ for attitude control of the

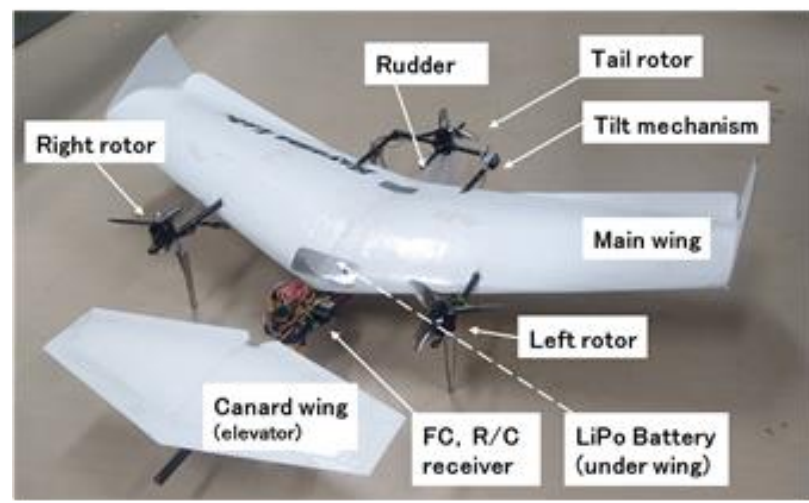

Fig. 5. Trial product of the proposed aircraft.

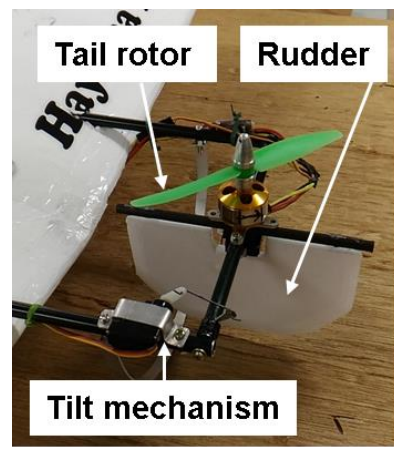

(a) Hovering

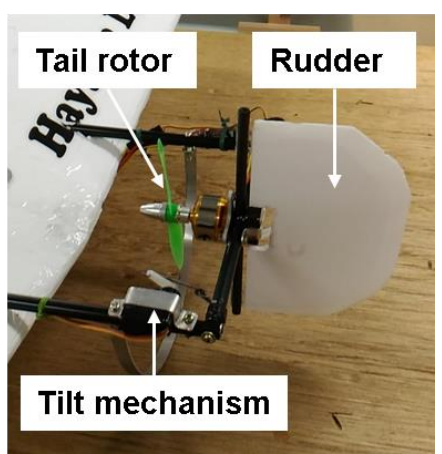

(b) Level flight
Fig. 6. Tilt of tail rotor and rudder.

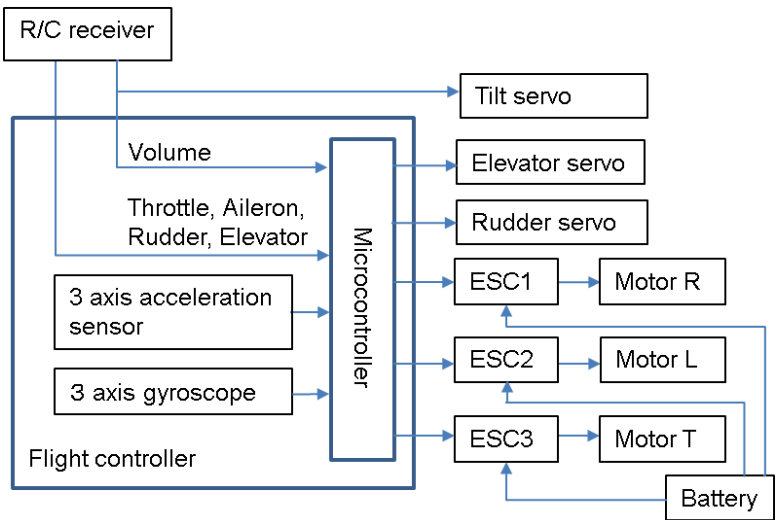

Fig. 7. Block diagram of the proposed aircraft.

Table 2. Custom FC setting for stabilization.

\begin{tabular}{|c|c|c|c|c|c|}
\hline & $\begin{array}{c}\text { Left } \\
\text { motor }\end{array}$ & $\begin{array}{c}\text { Right } \\
\text { motor }\end{array}$ & $\begin{array}{c}\text { Tail } \\
\text { motor }\end{array}$ & $\begin{array}{c}\text { Rudder } \\
\text { servo }\end{array}$ & $\begin{array}{c}\text { Elevator } \\
\text { servo }\end{array}$ \\
\hline Throttle & 127 & 127 & 127 & 0 & 0 \\
\hline Volume & -127 & -127 & 0 & 0 & 0 \\
\hline Roll & 127 & -127 & 0 & 0 & 0 \\
\hline Pitch & 64 & 64 & -127 & 0 & 127 \\
\hline Yaw & 0 & 0 & 0 & 127 & 0 \\
\hline
\end{tabular}

aircraft. The CC3D is commercially available flight controller. The control board is developed as open source/hardware project. The 3 -axis acceleration sensor and 3 -axis gyroscope are mounted in CC3D. The configuration of the attitude and flight control are possible by using a LibrePilot ${ }^{(10)}$. The CC3D is installed general tricopter firmware. Stabilization of hovering of the proposed aircraft is carried out by $\mathrm{CC} 3 \mathrm{D}$.

The FC has custom settings to transition to level flight as shown in table 2 . The value of 127 means maximum value for mixing channels. Tilting of the tail rotor is manually performed by volume control of the transmitter. The volume signal weakens the front rotors by the costom setting. At the same time, the PID control parameters of FC for stabilization of the aircraft are changed optimally according to the tilt angle of tail rotor.

\section{Test flight}

We conducted a test flight of the aircraft. After take-off vertically, the aircraft kept level and stable hovering was possible as shown in Fig. 8(a). The tail rotor tilt according to the volume operation, and the aircraft smoothly transitions from hovering to level flight. Fig. 8(b) shows a photograph when the aircraft transited to level flight. Operability was also gradually changed from multicopter to airplane. After tilted the rotor, a turning flight was possible by tilted rudder. As the airspeed increases, the 

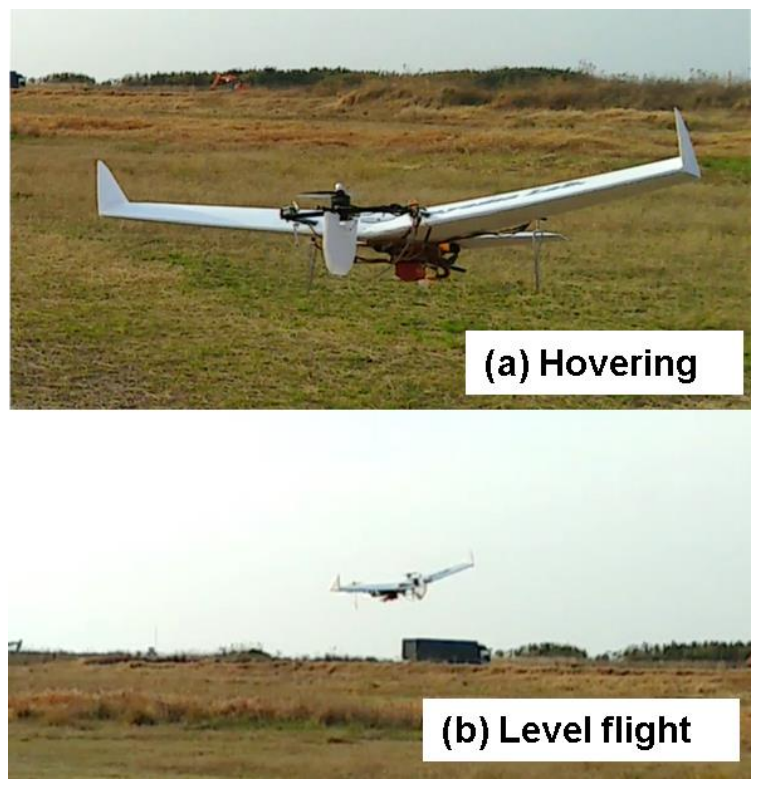

Fig. 8. Flight image of the proposed aircraft.

Table 3. Power consumption of proposed aircraft at difference angle of front rotors and fixed wing aircraft

\begin{tabular}{|c|c|}
\hline Flight attitude & Power consumption \\
\hline Hovering without tilt of rotor & $168 \mathrm{~W}$ \\
\hline Level flight with $90^{\circ}$ Tilt rotor & $48 \mathrm{~W}$ \\
\hline Fixed wing aircraft (Parrot Disco) & $40 \mathrm{~W}$ \\
\hline
\end{tabular}

aircraft rises the altitude by the lift of the wing. In order to keep the altitude, the throttle should to be down resulting in power saving.

In order to evaluate the power saving performance of the aircraft, the test flight had done with measuring the flight parameters. Table 3 shows the result of typical power consumption calculated from the battery voltage and current. The power consumption of well-known commercial fixed-wing aircraft (Parrot Disco ${ }^{(11)}$ ) of similar size is also listed in the table. Compared with the hovering, the aircraft during level flight can fly with less power by the lift of wing. The aircraft was able to fly with about $29 \%$ at $90^{\circ}$ tilted rotor.

\section{Conclusions}

The trial production of a new concept VTOL canard aircraft with tilt tail rotor was carried out. Continuous transition from hovering to level flight can be done by tilting the tail rotor. The lift of wing during level flight supported the weight of the aircraft, and its causes the reduction of power consumption. More detailed flight data and the advantages for the wide range observation will be demonstrated in near future.

\section{Acknowledgment}

This work is partially supported by Grant-in-Aid for Scientific Research (C) 20K11804.

\section{References}

(1) Jan Wendel, Oliver Meister, Christian Schlaile and Gert F. Trommer, "An integrated GPS/MEMS-IMU navigation system for an autonomous helicopter", Aerospace Science and Technology, Vol.10, Issue 6, 2006, pp.527-533.

(2) Koichi Kita, Atsushi Konno, and Masaru Uchiyama," Hovering Control of a Tail-Sitter VTOL Aerial Robot", Journal of Robotics and Mechatronics, Vol.21 No.2 pp. 277-283.

(3) Ryan X-13 Vertijet, https://en.wikipedia.org/ wiki/Ryan_X-13 Vertijet (accessed July 2, 2021).

(4) Jeremiah Gertler, "V-22 Osprey Tilt-Rotor Aircraft: Background and Issues for Congress", Congressional Research Service Reports, http://www.fas.org/sgp/ crs/weapons/RL31384.pdf, (accessed July 2, 2021).

(5) Wingcopter GmbH-Technology with a Purpose, https://wingcopter.com, (accessed July 2, 2021).

(6) QuadPlane Support, https://ardupilot.org/plane/docs/ quadplane-support.html, (accessed July 2, 2021).

(7) Kiyoteru Hayama, Hirofumi Ohtsuka and Hiroki Irie, "Trial Production of Variable Pitch Wing Attached Multicopter for Power Saving and Long Flight", Proc. of the 5th IIAE Int. Conf. on Intelligent Systems and Image Processing 2017 (ICISIP 2017), pp.36-41.

(8) Kiyoteru Hayama, Tomohiro Kudou, Kazuhiro Koshi, Hiroki Irie, "Trial Production of Kite Wing attached Tricopter with Tilt Front Rotors", Proc. of the 6th IIAE International Conference on Intelligent Systems and Image Processing 2019 (ICISIP 2019), pp.371-374

(9) CC Hardware Configuration-LibrePilot Documentation -Confluence,https://librepilot.atlassian.net/wiki/spaces/ LPDOC/pages/2818090/ (accessed July 2, 2021).

(10) LibrePilot-Open-Collavorative-Free, https://www. librepilot.org/site/index.html, (accessed June 6, 2019).

(11) Parrot disco Parrot DISCO FPV, https://www.youtube. com/watch?v=m1Fpq4inefg, (accessed July 2, 2021). 